\title{
Is blood pressure during the night more predictive of cardiovascular outcome than during the day?
}

Yan $\mathrm{Li}^{\mathrm{a}, \mathrm{b}}$, José Boggia ${ }^{\mathrm{c}}$, Lutgarde Thijs ${ }^{\mathrm{a}}$, Tine W. Hansen ${ }^{\mathrm{e}}$, Masahiro Kikuya ${ }^{\mathrm{g}}$, Kristina Björklund-Bodegård ${ }^{\text {h }}$, Tom Richart ${ }^{a}$, Takayoshi Ohkubog ${ }^{\text {, }}$ Tatiana Kuznetsova ${ }^{a}$, Christian Torp-Pedersen ${ }^{f}$, Lars Lind ${ }^{\mathrm{h}}$, Hans Ibsen ${ }^{f}$, Yutaka Imai ${ }^{9}$, Jiguang Wang ${ }^{b}$, Edgardo Sandoya ${ }^{d}$, Eoin O'Brien ${ }^{i}$ and Jan A. Staessen ${ }^{a}$ on behalf of the International Database on Ambulatory Blood Pressure Monitoring in relation to Cardiovascular Outcomes Investigators

The objective of this study was to investigate the prognostic significance of the ambulatory blood pressure (BP) during night and day and of the night-to-day BP ratio (NDR). We studied 7458 participants (mean age 56.8 years; $45.8 \%$ women) enrolled in the International Database on Ambulatory BP in relation to Cardiovascular Outcome. Using Cox models, we calculated hazard ratios (HR) adjusted for cohort and cardiovascular risk factors. Over 9.6 years (median), 983 deaths and 943 cardiovascular events occurred. Nighttime BP predicted mortality outcomes (HR, 1.18-1.24; $P<0.01)$ independent of daytime BP. Conversely, daytime systolic (HR, 0.84; $P<0.01)$ and diastolic BP (HR, 0.88; $P<0.05)$ predicted only noncardiovascular mortality after adjustment for nighttime BP. Both daytime BP and nighttime BP consistently predicted all cardiovascular events (HR, 1.11-1.33; $P<0.05)$ and stroke (HR, 1.21-1.47; $P<0.01)$. Daytime BP lost its prognostic significance for cardiovascular events in patients on antihypertensive treatment. Adjusted for the 24-h BP, NDR predicted mortality $(P<0.05)$, but not fatal combined with nonfatal events. Participants with systolic NDR of at least 1 compared with participants with normal NDR ( $\geq 0.80$ to $<0.90$ ) were older, at higher risk of death, but died at higher age. The predictive accuracy of the daytime and nighttime BP and the NDR depended on the disease outcome under study. The increased mortality in

\section{Introduction}

Numerous studies in populations and hypertensive patients have demonstrated that the 24-h ambulatory blood pressure (BP) carries more prognostic information in relation to cardiovascular outcomes than the office BP [1]. However, even in the presence of this evidence and the technical improvement over the past decades in the monitoring devices, most patients do not easily accept a BP recording over the whole day, and particularly at night. Clinicians are faced with deciding to what extent we need 24-h BP recording and whether the BP during specific periods, such as daytime or nighttime, has independent prognostic value. Outcomes from patients with higher NDR probably indicates reverse causality. Our findings support recording the ambulatory BP during the whole day. Blood Press Monit 13:145-147 (C) 2008 Wolters Kluwer Health | Lippincott Williams \& Wilkins.

Blood Pressure Monitoring 2008, 13:145-147

Keywords: ambulatory blood pressure monitoring, blood pressure, cardiovascular outcome

aDepartment of Cardiovascular Diseases, Division of Hypertension and Cardiovascular Rehabilitation, Studies Coordinating Centre, University of Leuven, Leuven, Belgium, ${ }^{\mathrm{b}}$ Center for Epidemiological Studies and Clinical Trials, Ruijin Hospital, Shanghai Jiaotong University School of Medicine, Shanghai, China, 'Department of Physiopathology, Manuel Quintela Hospital, University of the Republic, Montevideo, Uruguay, 'Spanish Primary Association of Mutual Aid, Montevideo, Uruguay, ${ }^{\mathrm{e} T h e}$ Research Center for Prevention and Health, ${ }^{f}$ Copenhagen University Hospital, Copenhagen, Denmark, 9The Tohoku University Graduate School of Pharmaceutical Sciences and Medicine, Sendai, Japan, ${ }^{\text {h}}$ The Section of Geriatrics, Department of Public Health and Caring Sciences, Uppsala University, Uppsala, Sweden and 'Conway Institute of Biomolecular and Biomedical Research, University College Dublin, Dublin, Ireland

Correspondence to Jan A. Staessen, MD, PhD, FESC, FAHA, Studies Coordinating Centre, Laboratory of Hypertension, University of Leuven, Campus Gasthuisberg, Herestraat 49, Box 702, B-3000 Leuven, Belgium Tel: + 3216347104 ; fax: + 3216347106 ; e-mail: jan.staessen@med.kuleuven.be

Received 9 October 2007 Revised 9 October 2007 Accepted 10 October 2007

previous studies have usually shown that an elevated nocturnal $\mathrm{BP}$ is a harbinger of an unfavorable outcome [2-4]. However, the interpretation of these studies is often difficult, because of restriction of follow-up to mortality, the confounding effects of antihypertensive drug treatment, and/or the use of different diagnostic thresholds for the night-to-day BP ratio (NDR). The purpose of this paper is to summarize our recent findings [5] on the prognostic accuracy of daytime versus nighttime BP and the NDR in 7458 participants enrolled in prospective population studies in Denmark [2], Belgium [6], Japan [3], Sweden [4], Uruguay [7], and China [8]. 


\section{Methods}

We constructed the International Database on Ambulatory BP Monitoring in relation to Cardiovascular Outcomes according to the previously published protocol [9]. The same SAS macro processed all ambulatory recordings. We defined daytime as the interval ranging from 10.00 to $20.00 \mathrm{~h}$ in Europeans [2,6] and South Americans [7] and from 08.00 to $18.00 \mathrm{~h}$ in Asians [3,8]. The corresponding nighttime intervals ranged from midnight to $06.00 \mathrm{~h}$ and from 22.00 to $04.00 \mathrm{~h}$, respectively. Normal dipping was NDR ranging from less than 0.90 to 0.80 inclusive. We labeled the other categories of dipping status as extreme $(<0.80)$, decreased (from $\geq 0.90$ to $<1.00)$ and reverse $(>1.00)$. Vital status and incidence of fatal and nonfatal diseases were ascertained from the appropriate sources in each country, as described in detail in previous publications [2-4,10]. For database management and statistical analysis, we used SAS software, version 9.1.3 (SAS Institute, Cary, North Carolina, USA).

\section{Results}

\section{Baseline characteristics}

The 7458 participants included 3416 women (45.8\%) and 3436 patients with hypertension on conventional BP measurement (46.1\%), of whom 1637 (47.6\%) patients were taking BP-lowering drugs. Mean (SD) age was 56.8 (13.9) years. The median follow-up was 9.6 years (5th-95th percentile interval, 2.5-13.7 years). During the follow-up, 983 participants died (14.1 per 1000 personyears) and 943 experienced a fatal or nonfatal cardiovascular complication (13.6 per 1000 person-years). Mortality included 387 cardiovascular and 560 noncardiovascular deaths and 36 deaths from unknown cause.

\section{Risk associated with daytime and nighttime blood pressure}

After adjustment for cohort, sex, age, body mass index, smoking and drinking habits, serum total cholesterol, history of cardiovascular disease, diabetes mellitus, and antihypertensive drug treatment, nighttime BP predicted all mortality outcomes [hazard ratio (HR), 1.09-1.29;
$P<0.05]$, whereas daytime BP predicted total and cardiovascular mortality (HR, 1.09-1.29; $P<0.05)$, but not death from noncardiovascular causes (HR, 0.96-0.97; $P>0.31)$. When additionally adjusted for daytime $\mathrm{BP}$, the nighttime $\mathrm{BP}$ remained a significant predictor of all mortality outcomes (Table 1). In contrast, in the presence of nighttime BP, the daytime BP lost significance for total and cardiovascular mortality, but became significant for noncardiovascular mortality, higher levels being associated with lower relative risk (Table 1).

With adjustments applied for cohort and risk factors, the daytime and nighttime systolic and diastolic BPs consistently predicted all cardiovascular events (HR, 1.251.33; $P<0.0001)$ and fatal and nonfatal stroke (HR, 1.39-1.47; $P<0.0001)$. When additionally adjusted for nighttime $\mathrm{BP}$, the systolic daytime $\mathrm{BP}$ lost its prognostic significance for cardiac events, whereas the diastolic daytime BP became nonsignificant for cardiac and coronary events (Table 1). Conversely, in the presence of the daytime BP, the systolic and diastolic nighttime levels no longer predicted coronary events.

Addition of the interaction terms between treatment status and the daytime and nighttime BPs to the models considerably improved the fit (log likelihood ratio test; $P<0.0001)$. Nighttime systolic BP remained as the significant predictor $(P<0.05)$ for total mortality and cardiovascular events irrespective of treatment status. However, daytime systolic BP predicted cardiovascular events only in untreated participants (HR, 1.30; $P<0.0001)$, but not in treated patients (HR, 1.01; $P=0.87)$.

\section{Risk associated with night-to-day BP ratio}

In continuous analyses, the systolic and diastolic NDR significantly predicted total, noncardiovascular and cardiovascular mortality (HR, 1.13-1.16; $P<0.01)$ in models adjusted for cohort and risk factors. This was also the case when additionally adjusted for $24 \mathrm{~h}$ BP (Table 1). In

Table 1 Adjusted standardized hazard ratios for daytime and nighttime blood pressure and systolic night-to-day ratio

\begin{tabular}{|c|c|c|c|c|c|c|}
\hline \multirow[b]{2}{*}{ Label } & \multicolumn{2}{|c|}{ Daytime blood pressure } & \multicolumn{2}{|c|}{ Nighttime blood pressure } & \multicolumn{2}{|c|}{ Night-to-day blood pressure ratio } \\
\hline & Systolic & Diastolic & Systolic & Diastolic & Systolic & Diastolic \\
\hline Total mortality & $0.94(0.87-1.03)$ & $0.94(0.87-1.03)$ & $1.22(1.13-1.31)^{\S}$ & $1.20(1.11-1.30)^{\S}$ & $1.13(1.07-1.19)^{\S}$ & $1.12(1.06-1.19)^{\ddagger}$ \\
\hline Cardiovascular & $1.11(0.98-1.27)$ & $1.07(0.94-1.22)$ & $1.22(1.09-1.36)^{\ddagger}$ & $1.24(1.10-1.40)^{\ddagger}$ & $1.08(0.99-1.17)$ & $1.10(1.00-1.21)^{*}$ \\
\hline Noncardiovascular & $0.84(0.75-0.94)^{\dagger}$ & $0.88(0.79-0.98) *$ & $1.21(1.10-1.33)^{\ddagger}$ & $1.18(1.06-1.31)^{\dagger}$ & $1.16(1.08-1.25)^{\S}$ & $1.14(1.05-1.23)^{\dagger}$ \\
\hline $\begin{array}{l}\text { All cardiovascular } \\
\text { events }\end{array}$ & $1.16(1.07-1.26)^{\ddagger}$ & $1.11(1.02-1.20)^{*}$ & $1.21(1.12-1.30)^{\S}$ & $1.20(1.11-1.30)^{\S}$ & $1.05(0.98-1.11)$ & $1.07(1.00-1.13)^{*}$ \\
\hline Stroke & $1.27(1.13-1.43)^{\ddagger}$ & $1.21(1.07-1.37)^{\dagger}$ & $1.23(1.11-1.37)^{\ddagger}$ & $1.24(1.10-1.39)^{\ddagger}$ & $1.02(0.94-1.11)$ & $1.04(0.95-1.14)$ \\
\hline Cardiac & $1.11(0.99-1.24)$ & $1.04(0.93-1.17)$ & $1.17(1.06-1.29)^{\dagger}$ & $1.15(1.04-1.28)^{\dagger}$ & $1.05(0.97-1.14)$ & $1.07(0.98-1.16)$ \\
\hline Coronary & $1.19(1.04-1.36)^{*}$ & $1.11(0.98-1.27)$ & $1.05(0.93-1.18)$ & $1.08(0.96-1.22)$ & $0.97(0.89-1.07)$ & $1.00(0.91-1.11)$ \\
\hline
\end{tabular}

Values are standardized hazard ratios (95\% confidence intervals), which express the risk per SD increase in the blood-pressure variables. Systolic/diastolic SDs were $15.52 / 9.33 \mathrm{mmHg}$ and $15.53 / 9.25 \mathrm{mmHg}$ for the day and night blood pressures, respectively, and 0.08/0.09 for the night-to-day blood pressure ratio. All hazard ratios were adjusted for cohort, sex, age, body mass index, smoking and drinking, serum cholesterol, history of cardiovascular disease, diabetes mellitus, and antihypertensive drug treatment. The daytime blood pressure was additionally adjusted for the nighttime blood pressure (and vice versa), and the night-to-day ratio was additionally adjusted for the 24-h blood pressure. Significance of the hazard ratios: ${ }^{*} P<0.05,{ }^{\dagger} P<0.01,{ }^{\ddagger} P<0.001$, and ${ }^{\S} P<0.0001$. 
contrast to mortality, the systolic and diastolic NDR did not behave as consistent predictors of fatal combined with nonfatal events. In fully adjusted models, the systolic NDR was nonsignificant for all combined fatal and nonfatal outcomes under study. The diastolic NDR was only a significant predictor of all cardiovascular outcomes, but not of other combined fatal and nonfatal events (Table 1 ).

In categorical analyses, with adjustments applied for cohort, cardiovascular risk factors and the $24 \mathrm{~h}$ BP, reversed dipping were associated with increased risk of death from any cause (HR, 1.56; $P<0.0001)$ and the composite of all fatal and nonfatal cardiovascular complications (HR, 1.30; $P<0.05)$. Compared with participants with a normal NDR, reverse dippers were not only older (55.2 vs. 62.9 years; $P<0.0001$ ) at enrollment, but also died at a higher age $(74.1$ vs. 78.2 years; $P<0.0001)$. Both cardiovascular $(75.9$ vs. 78.9 years; $P=0.002)$ and noncardiovascular ( 73.6 vs. 77.5 years; $P=0.001$ ) mortality contributed to the latter observation.

\section{Conclusion}

In this study of a large cohort, the predictive accuracy of the daytime and nighttime BPs and the NDR depended on the disease outcome under study. The increased mortality in patients with higher NDR probably indicates reverse causality. Our findings support, therefore, recording the ambulatory BP during the whole day in clinical practice.

\section{References}

1 O'Brien E, Asmar R, Beilin L, Imai Y, Mancia G, Mengden T, et al.; European Society of Hypertension Working Group on Blood Pressure Monitoring. Practice guidelines of the European Society of Hypertension for clinic, ambulatory and self blood pressure measurement. J Hypertens 2005; 23:697-701.

2 Hansen TW, Jeppesen J, Rasmussen F, Ibsen H, Torp-Pedersen C. Ambulatory blood pressure monitoring and mortality: a population-based study. Hypertension 2005; 45:499-504.

3 Ohkubo T, Hozawa A, Yamaguchi J, Kikuya M, Ohmori K, Michimata M, et al. Prognostic significance of the nocturnal decline in blood pressure in individuals with and without high 24-h blood pressure: the Ohasama study. J Hypertens 2002; 20:2183-2189.

4 Ingelsson E, Björklund K, Lind L, Ärnlöv J, Sundström J. Diurnal blood pressure pattern and risk of congestive heart failure. J Am Med Assoc 2006; 295:2859-2866.

5 Boggia J, Li Y, Thijs L, Hansen TW, Kikuya M, Björklund-Bodegård K, et al.; on behalf of the International Database on Ambulatory blood pressure monitoring in relation to Cardiovascular Outcomes (IDACO) Investigators. Prognostic accuracy of day versus night ambulatory blood pressure: a cohort study. Lancet 2007; 370:1221-1231.

6 Staessen JA, Bieniaszewski L, O'Brien ET, Imai Y, Fagard R. An epidemiological approach to ambulatory blood pressure monitoring: the Belgian population study. Blood Press Monit 1996; 1:13-26.

7 Schettini C, Bianchi M, Nieto F, Sandoya E, Senra H; Hypertension Working Group. Ambulatory blood pressure: normality and comparison with other measurements. Hypertension 1999; 34 (Part 2):818-825.

8 Li Y, Wang JG, Gao HF, Nawrot T, Wang GL, Qian YS, et al. Are published characteristics of the ambulatory blood pressure generalizable to rural Chinese? The JingNing population study. Blood Press Monit 2005; 10:125-134.

9 Thijs L, Hansen TW, Kikuya M, Bjorklund-Bodegard K, Li Y, Dolan E, et al.; IDACO Investigators. The International Database of Ambulatory Blood Pressure in relation to Cardiovascular Outcome (IDACO): protocol and research perspectives. Blood Press Monit 2007; 12:255-262.

10 Li Y, Thijs L, Kuznetsova T, Zagato L, Struijker-Boudier H, Bianchi G, Staessen JA. Cardiovascular risk in relation to a-adducin Gly460Trp polymorphism and systolic pressure. A prospective population study. Hypertension 2005; 46:527-532. 